\title{
Sharp bounds on sufficient-cause interactions under the assumption of no redundancy
}

\author{
Wen-Chung Lee
}

\begin{abstract}
Background: Sufficient-cause interaction is a type of interaction that has received much attention recently. The sufficient component cause model on which the sufficient-cause interaction is based is however a non-identifiable model. Estimating the interaction parameters from the model is mathematically impossible.

Methods: In this paper, I derive bounding formulae for sufficient-cause interactions under the assumption of no redundancy.

Results: Two real data sets are used to demonstrate the method ( $R$ codes provided). The proposed bounds are sharp and sharper than previous bounds.

Conclusions: Sufficient-cause interactions can be quantified by setting bounds on them.

Keywords: Sufficient component cause model, Epidemiologic methods, Causal inference, Interaction, Identifiability
\end{abstract}

\section{Background}

A common aim of many observational studies is to identify risk factors for disease. Once risk factors have been identified, researchers will often be interested in knowing whether any two factors can interact in causing the disease. 'Sufficient-cause interaction' (also referred to as 'synergism, 'causal co-action', 'causal mechanistic interaction', or simply 'mechanistic interaction') is a type of interaction that has received much attention recently [1-11] and is based on Rothman's sufficient component cause model $[12,13]$. The model posits that the causation of disease can be through any one of many different mechanisms or pathways. A mechanism/pathway requires several different component causes to operate, hence it is also called a 'causal pie'. If two factors participate in the same causal pie, then a sufficientcause interaction can be said to exist between them.

If the monotonicity assumption is not imposed [14-18], the sufficient component cause model in its general form is over-parameterized and non-identifiable. That is, the total number of model parameters exceeds the total degrees of

Correspondence: wenchung@ntu.edu.tw

Research Center for Genes, Environment and Human Health and Institute of Epidemiology and Preventive Medicine, College of Public Health, National Taiwan University, Rm. 536, No. 17, Xuzhou Rd., Taipei 100, Taiwan freedom the data can offer. For example, two binary risk factors mean the data can offer at most four degrees of freedom (four different exposure profiles) but the model has a total of nine parameters, each corresponding to one of the nine possible causal-pie classes (one 'all-unknown' class unrelated to either factor, two main-effect classes for each factor, and four two-factor interaction classes). [If the monotonicity assumption is imposed on the two factors, the number of causal-pie classes reduces to four (one 'all-unknown' class unrelated to either factor, one main-effect class for each factor, and one two-factor interaction class), and the model becomes identifiable.] Researchers recently found ways to circumvent the non-identifiability problem and have developed methods to test for sufficient-cause interactions without imposing the monotonicity assumption [1-11]. It is however mathematically impossible to estimate the interaction parameters from a truly non-identifiable sufficient component cause model. At best, bounds can be set.

In this paper, I derive the bounding formulae for sufficient-cause interactions under the assumption of no redundancy $[6-11,19]$. $R$ codes for all computations are provided for convenience and the method is demonstrated with two real datasets. The proposed bounds will also be shown to be sharp and sharper than previous bounds [20]. 


\section{Methods}

\section{Notations and definitions}

This paper closely follows the notations used in previous studies [6-11]. Here, we are interested in the relationship between two exposures and a binary outcome (e.g., disease/no disease). We assume a population is studied from time 0 to $T$. The two exposures $\left(X_{1}\right.$ and $\left.X_{2}\right)$ can have arbitrarily many levels (a total of $L_{1} \geq 2$ and $L_{2} \geq 2$, respectively). We assume that the exposure profile for a person does not change over time during the study period and is represented by profile $=x_{1}, x_{2}$, with $x_{1} \in$ $\left\{1, \ldots, L_{1}\right\}$ and $x_{2} \in\left\{1, \ldots, L_{2}\right\}$ We assume that there is no loss to follow up and competing death during this study period. Let $D=1$ represent disease occurrence in $(0, T)$, and $D=0$, otherwise. We assume $D$ is known but the exact time of disease occurrence, if ever, is unknown to researchers. ( $D$ is a binary outcome within a defined period, not a time-to-event outcome.) It is assumed that there is no confounding, selection bias or measurement error in the study. The associations between the two exposures and the disease should reflect the genuine causal effects of the exposures on the disease.

While there is only a total of $L_{1} \times L_{2}$ exposure profiles, there is a total of $\left(L_{1}+1\right) \times\left(L_{2}+1\right)$ different causal-pie classes, including one all-unknown class, $L_{1}+L_{2}$ maineffect classes, and $L_{1} \times L_{2}$ interaction classes. (Figure 1 in Lee's paper [7] depicts $(2+1) \times(2+1)=9$ causal-pie classes in total for two binary exposures.) The causal-pie classes can be represented by class $=c_{1}, c_{2}$, with $c_{1} \in\left\{*, 1, \ldots, L_{1}\right\}$ and $c_{2} \in\left\{*, 1, \ldots, L_{2}\right\}$. Note that here we introduce a null notation *, such that a class contains for $k=1,2$, " $X_{k}=c_{k}$ " as one of its component causes if $c_{k} \neq *$, and does not involve $X_{k}$ whatsoever if $c_{k}=*$. For example, the all-unknown class involving neither $X_{1}$ nor $X_{2}$ is represented by class $={ }^{*}$,*; the main-effect classes are represented by class $=c_{1},{ }^{*}$ with $c_{1} \neq{ }^{*}$ for $X_{1}$-only classes, and class $={ }^{*}, c_{2}$ with $c_{2} \neq{ }^{*}$ for $X_{2}$ only classes; and the interaction classes are represented by class $=c_{1}, c_{2}$ with $\mathrm{c}_{1} \neq *$ and $\mathrm{c}_{2} \neq *$.

The sufficient component cause model is partly deterministic and partly stochastic. The presence of risk factor(s) alone is not sufficient for the disease. Only when all unknown components (complement causes) also appear can the sufficient cause become complete and the disease occur. We let $U_{c_{1}, c_{2}}=1$ represent the arrival of the unknown components of the class $=c_{1}, c_{2}$ causal-pie class in $(0, T)$, and $U_{c_{1}, c_{2}}=0$, otherwise, for $c_{1} \in\left\{, 1, \ldots, L_{1}\right\}$ and $c_{2} \in\left\{, 1, \ldots, L_{2}\right\}$.

\section{Cumulative disease risk, cumulative completion risk, and relative prevalence}

Let Risk $^{\text {profile }=x_{1}, x_{2}}$ denote the cumulative disease risk in $(0, T)$ for people in the population with profile $=x_{1}, x_{2}$, that is, $\operatorname{Pr}\left(D=1 \mid X_{1}=x_{1}, X_{2}=x_{2}\right)$. Let Risk class $=i, j_{\text {denote }}$ the cumulative completion risk in $(0, T)$ for a specific class $=i, j$ sufficient-cause interaction, that is, $\operatorname{Pr}\left(U_{i j}=1\right)$ for the specific $i \in\left\{1, \ldots, L_{1}\right\}$ and $j \in\left\{1, \ldots, L_{2}\right\}$. Let Riskclass $=$ int denote the cumulative completion risk in $(0, T)$ for the global sufficient-cause interaction (sufficient-

cause interaction regardless of classes), that is, $\operatorname{Pr}$ $\left[\underset{\substack{i \in\left\{1, \ldots, L_{1}\right\} \\ j \in\left\{1, \ldots, L_{2}\right\}}}{\cup}\left(U_{i j}=1\right)\right]$. Let Risk $_{\text {class }=\text { any }}$ denote the cumulative completion risk over $(0, T)$ for any class (all-unknown, main-effect, or interaction), that is, $\operatorname{Pr}\left[\underset{\substack{i \in\left\{*, 1, \ldots, L_{1}\right\}, j \in\left\{*, 1, \ldots, L_{2}\right\}}}{\cup}\left(U_{i j}=1\right)\right]$, or equivalently, the proportion of those excluding the 'immune' persons in the study population during the study period. (An immune person is one who will not contract the disease during the study period, no matter what exposure profile he/she might contrary-to-fact assume.)

If the disease is rare we would always expect the above cumulative completion risks (or period prevalence, since these are defined for subjects in the study population over the study period) to be close to 0 . To be informative for interactions for rare diseases, here we follow Sjölander et al.'s suggestion [20] to define the relative prevalence $(\mathrm{RP})$ for the specific sufficient-cause interac-

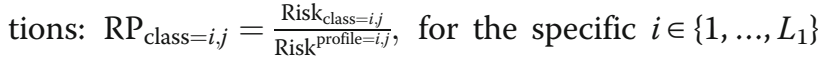
and $j \in\left\{1, \ldots, L_{2}\right\}$. In addition, we also define a relative prevalence for the global sufficient-cause interaction: $\mathrm{RP}_{\text {class }=\text { int }}=\frac{\text { Risk }_{\text {class }=\text { int }}}{\text { Risk }_{\text {class=any }}}$. Note that specific and global RPs assume different denominators.

\section{The no-redundancy assumption}

The no-redundancy assumption is a Poisson-like assumption which dictates there can only be at most one arrival event of the unknown components (at most one class of sufficient causes that can be completed) in a sufficiently short time interval for each and every subject in the population [19]. In other words, there are at most $\left(L_{1}+1\right) \times\left(L_{2}+1\right)+1$ causal response types in a very short time interval, with each of the $\left(L_{1}+1\right) \times\left(L_{2}+1\right)$ types corresponding to exactly one causal-pie class, plus an additional one for the immune type. The table in Lee's paper [6] enumerates the total $(2+1) \times(2+1)+1=$ 10 causal response types for two binary exposures under the no-redundancy assumption. By comparison, the conventional potential outcome model (without the no-redundancy assumption) would have a total of $2^{L_{1} \times L_{2}}$ causal response types, and $2^{2 \times 2}=16$ for two binary exposures. 
The no-redundancy assumption is a relatively weak assumption that can still hold true even if there is a strong dependency in the arrival events. Note that no redundancy is specified only with respect to an infinitesimally short time interval. It says nothing about the entire follow-up period and can therefore also hold true even for non-rare diseases (diseases with high Risk ${ }^{\text {profile }=i, j}$ for $i \in\left\{1, \ldots, L_{1}\right\}$ and $\left.j \in\left\{1, \ldots, L_{2}\right\}\right)$. Several sufficient-cause interaction tests had previously been developed under this assumption [6-11].

\section{Bounds on sufficient-cause interactions under the no-redundancy assumption}

In Additional file 1, I derive the bounds on sufficientcause interactions under the no-redundancy assumption. For the specific sufficient-cause interactions, the bounds are (LB in superscript for lower bound; UB for upper bound):

$$
\begin{aligned}
\operatorname{Risk}_{\text {class }=i, j}^{\mathrm{LB}}=1- & \min _{\substack{\left.i^{\prime} \neq i\right) \in\left\{1, \ldots, L_{1}\right\} \\
\left(j^{\prime} \neq j\right) \in\left\{1, \ldots, L_{2}\right\}}} \\
& \left\{\frac{1-\text { Risk }^{\text {profile }=i, j}}{\left(1-\text { Risk }^{\left.{\text {profile }=i^{\prime}, j}_{j}\right) \times\left(1-\text { Risk }^{\text {profile }=i, j^{\prime}}\right)}, 1\right.}\right\},
\end{aligned}
$$

$$
\begin{aligned}
& \operatorname{Risk}_{\text {class }=i, j}^{\mathrm{UB}}=\operatorname{Risk}^{\text {profile }=i, j}, \\
& \operatorname{RP}_{\text {class }=i, j}^{\mathrm{LB}}=\frac{\operatorname{Risk}_{\text {class }=i, j}^{\mathrm{LB}}}{\operatorname{Risk}^{\mathrm{profile}=i, j}},
\end{aligned}
$$

and

$$
\mathrm{RP}_{\text {class }=i, j}^{\mathrm{UB}}=1,
$$

respectively, for the specific $i \in\left\{1, \ldots, L_{1}\right\}$ and $j \in\left\{1, \ldots, L_{2}\right\}$. For the global sufficient-cause interaction, the bounds are:

$$
\begin{aligned}
& \text { Risk }_{\text {class }=\text { int }}^{\mathrm{LB}}=1-\min _{\text {permutations of }\left(u_{1}, \ldots, u_{L_{1}}\right)} \\
& \text { and permutations of }\left(v_{1}, \ldots, v_{L_{2}}\right) \\
& \left\{\prod_{i=1}^{L_{1}} \prod_{j=1}^{L_{2}}\left(1-\text { Risk }^{\text {profile }=i, j}\right)^{u_{i} \times v_{j}}, 1\right\}, \\
& \operatorname{Risk}_{\text {class }=\text { int }}^{\mathrm{UB}}=1-\prod_{i=1}^{L_{1}} \prod_{j=1}^{L_{2}}\left(1-\operatorname{Risk}^{\mathrm{profile}=i, j}\right) \text {, } \\
& \mathrm{RP}_{\text {class=int }}^{\mathrm{LB}}=\frac{\operatorname{Risk}_{\text {class=int }}^{\mathrm{LB}}}{1-\prod_{i=1}^{L_{1}} \prod_{j=1}^{L_{2}}\left(1-\text { Risk }^{\mathrm{profile}=i, j}\right)},
\end{aligned}
$$

$$
\mathrm{RP}_{\text {class=int }}^{\mathrm{UB}}=1,
$$

respectively. $\left[\right.$ Risk $_{\text {class }=\text { int }}^{\mathrm{LB}}$ involves the use of 'contrast coefficients'. The contrast coefficients for $X_{1}$, $\left(u_{1}, \ldots, u_{L_{1}}\right)$, contains as its elements an equal number of ' +1 ' and ' -1 ' if $L_{1}$ is an even number, and exactly one ' 0 ' and an equal number of ' +1 ' and ' -1 ' for the remaining elements if otherwise. The contrast coefficients for $X_{2},\left(v_{1}, \ldots, v_{L_{2}}\right)$, are similarly constructed.]

When both exposures are binary, the lower bound formula is simplified considerably. Formula (1) becomes

$$
\operatorname{Risk}_{\text {class }-i j}^{\mathrm{LB}}=1-\min \left\{\frac{1-\text { Risk }^{\text {profile }=i, j}}{\left(1-\text { Risk }^{\text {proffle }=3-i, j}\right) \times\left(1-\text { Risk }^{\text {profile }=i, 3-j}\right)}, 1\right\},
$$

for $i, j \in\{1,2\}$. Formula (5) becomes

$$
\text { Risk }_{\text {class=int }}^{\mathrm{LB}}=1-\min \left(\text { PRISM, }_{\text {PRISM }}^{-1}\right),
$$

where PRISM $=\frac{\left(1-\text { Risk }^{\text {profile }=2,1}\right) \times\left(1-\text { Risk }^{\text {profile }=1,2}\right)}{\left(1-\text { Risk }^{\text {profile }=2,2}\right) \times\left(1-\text { Risk }^{\text {profile }=1,1}\right)}$ is the 'peril ratio index of synergy based on multiplicativity' [7].

\section{Case-control study for rare diseases}

For a rare disease with exceedingly low risks, we have

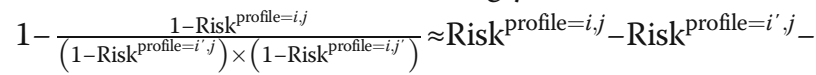
$\operatorname{Risk}^{\text {profile }=i, j^{\prime}}$ for $\left(i^{\prime} \neq i\right) \in\left\{1, \ldots, L_{1}\right\}$ and $\left(j^{\prime} \neq j\right) \in\left\{1, \ldots, L_{2}\right\}$, $1-\prod_{i=1}^{L_{1}} \prod_{j=1}^{L_{2}}\left(1-\text { Risk }^{\mathrm{profile}=i, j}\right)^{u_{i} \times v_{j}} \approx \sum_{i=1}^{L_{1}} \sum_{j=1}^{L_{2}} u_{i} \times v_{j} \times$ Risk $^{\mathrm{profile}=i, j}$ , and $1-\prod_{i=1}^{L_{1}} \prod_{j=1}^{L_{2}}\left(1-\right.$ Risk $\left.^{\text {profile }=i, j}\right) \approx \sum_{i=1}^{L_{1}} \sum_{j=1}^{L_{2}}$ Risk $^{\text {profile }=i, j}$. Therefore, the lower bounds on the relative prevalence of sufficient-cause interactions are approximately

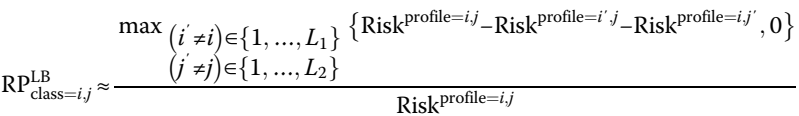

$$
\begin{aligned}
& \approx \frac{\max \left(\begin{array}{c}
\left.i^{\prime} \neq i\right) \in\left\{1, \ldots, L_{1}\right\} \\
\left.j^{\prime} \neq j\right) \in\left\{1, \ldots, L_{2}\right\}
\end{array}\right.}{\mathrm{OR}^{\text {proffle }=i, j}}
\end{aligned}
$$

and for the specific $i \in\left\{1, \ldots, L_{1}\right\}$ and $j \in\left\{1, \ldots, L_{2}\right\}$, and 


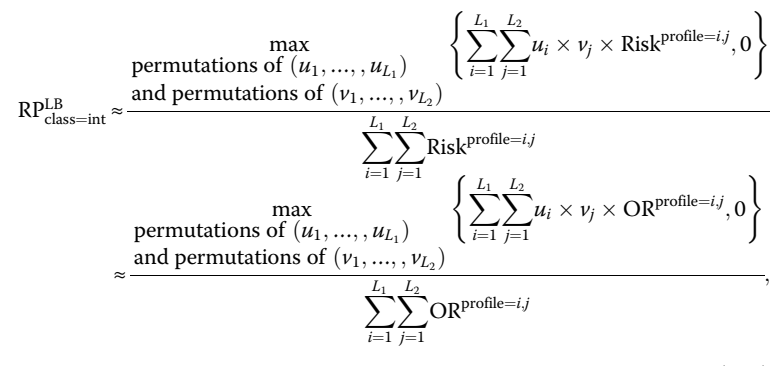

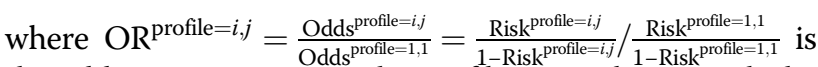
the odds ratio comparing the profile $=i, j$ subjects with the profile $=1,1$ subjects. These bounds are functions of odds ratios and can therefore be estimated directly from a casecontrol study conducted in the study population.

When both exposures are binary, the bounds reduce to

$$
\mathrm{RP}_{\text {class }=i, j}^{\mathrm{LB}} \approx \frac{\max \left\{\mathrm{OR}^{\mathrm{profile}=i, j}-\mathrm{OR}^{\mathrm{profile}=3-i, j}-\mathrm{OR}^{\mathrm{profile}=i, 3-j}, 0\right\}}{\mathrm{OR}^{\text {profile }=i, j}},
$$

for the specific $i, j \in\{1,2\}$, and

$$
\mathrm{RP}_{\text {class=int }}^{\mathrm{LB}} \approx \frac{|\mathrm{RERI}|}{\mathrm{OR}^{\text {profile=2,2}}+\mathrm{OR}^{\text {profile=2,1 }}+\mathrm{OR}^{\text {profile=1,2 }}+1,}
$$

where $\quad$ RERI $=\mathrm{OR}^{\text {profile }=2,2}-\mathrm{OR}^{\text {profile }=2,1}-\mathrm{OR}^{\text {profile }=1,2}$ +1 is the 'relative excess risk due to interaction' in terms of odds ratios [1-5].

Additional file 2 presents two functions written in $\mathrm{R}$ code: 'bounds.cohort' for cohort data and 'bounds.cscn' for case-control data. Input the data as the argument and the functions will output the various bounds on sufficient-cause interactions. Additionally, the functions also automatically perform 10,000 bootstrap replications to calculate a $95 \%$ lower confidence limit for a lower bound and a 95\% upper confidence limit for an upper bound.

\section{Results}

\section{Example 1. A cohort study of hypertension risk}

The data of a cohort study on hypertension risk (taken directly from Example 3 in Zou's paper [21]) is analyzed here as an example. The cohort study assesses the effects of body mass index (BMI, coded as 1 if BMI $\geq 25 \mathrm{~kg} / \mathrm{m}^{2}$ and 0 if otherwise) and age (coded as 1 if age $\geq 40$ years and 0 if otherwise) on hypertension (coded as 1 if diastolic blood pressure $\geq 90 \mathrm{mmHg}$ and 0 if otherwise). We assume that there is no confounding, selection bias or measurement error in the study and that the followup is $100 \%$ complete.

Table 1 presents the bounds and their 95\% bootstrapped confidence limits for sufficient-cause interactions between BMI and age. The lower bounds for the (high BMI, old age)-specific sufficient-cause interaction are greater than zero $(0.0411$ for the cumulative completion risk; 0.1509 for the relative prevalence), but do not achieve statistical significance (as judged from their 95\% lower confidence limits which are both zero). As for the global sufficient-cause interactions, the lower bounds are 0.0830 (cumulative completion risk) and 0.1758 (relative prevalence), respectively, and are both significantly greater than zero. The upper bound for the cumulative completion risk of the global sufficient-cause interaction is 0.4718 with an upper $95 \%$ confidence limit of 0.4993 .

\section{Example 2. A case-control study on lung cancer risk}

Zhang et al.'s case-control data (directly taken from Table 4 in reference [22]) is analyzed here as the second example. The study examines the gene-gene interactions between two DNA base excision repair genes on lung cancer risk: the ADPRT (adenosine diphosphate ribosyltransferase) Val762Ala polymorphism and the XRCC1 (X-ray repair cross-complementing group 1) Arg366Gln polymorphism (both having three genotypes). The raredisease assumption is invoked here (For lung cancer, the assumption is tenable). In addition, we assume geneenvironment independence [10] such that unmeasured environmental factors, no matter what they may be, cannot confound the genetic effects of the two studied genes.

Table 2 presents the lower bounds and the 95\% lower limits for sufficient-cause interactions between these two genes. The lower bound of the relative prevalence for the $(A D P R T=A l a / A l a, X R C C 1=G \ln / G l n)$-specific sufficientcause interaction is greater than zero $(0.5221)$ but does not achieve statistical significance. The lower bound of the relative prevalence for the global $A D P R T$ $X R C C 1$ interaction is 0.2471 and is significantly greater than zero (as judged from its 95\% lower confidence limit which is 0.0784).

\section{Discussion}

Public health researchers have long sought a way to quantify sufficient-cause interactions using only the observational data at hand. Due to the non-identifiability problem, a sufficient-cause interaction can be tested but unfortunately not estimated. We are therefore provided with a very limited piece of information (of whether or not a sufficient-cause interaction is statistically significant), which falls far short of quantification. By setting bounds on sufficient-cause interactions (as demonstrated in the two examples in this paper), we can finally make some actual (if not exact) quantifications of such interactions.

Additional file 3 shows that the bounding formulae we presented in this paper produce 'sharp' bounds, i.e., bounds that are attainable. Previously, Sjölander et al. [20] derived an assumption-free lower bound 
Table 1 Bounds on sufficient-cause interactions in a cohort study on hypertension risk (Example 1)

\begin{tabular}{|c|c|c|c|c|c|c|c|c|c|}
\hline & \multirow[t]{2}{*}{$\begin{array}{l}\text { Case } \\
\text { number }\end{array}$} & \multirow[t]{2}{*}{ Population } & \multirow[t]{2}{*}{ Risk } & \multicolumn{4}{|c|}{$\begin{array}{l}\text { Cumulative completion risk of } \\
\text { sufficient-cause interaction }\end{array}$} & \multicolumn{2}{|c|}{$\begin{array}{l}\text { Relative prevalence of } \\
\text { sufficient-cause interaction }\end{array}$} \\
\hline & & & & $95 \% \mathrm{LCL}^{\mathrm{c}}$ & $\mathrm{LB}^{\mathrm{C}}$ & $U B^{C}$ & $95 \% \mathrm{UCL}^{\mathrm{c}}$ & $95 \% \mathrm{LCL}^{\mathrm{c}}$ & $\mathrm{LB}^{\mathrm{C}}$ \\
\hline \multicolumn{10}{|c|}{ Specific (BMIl, age $\left.{ }^{b}\right)$} \\
\hline (low, young) & 79 & 1810 & 0.0437 & 0.0000 & 0.0000 & 0.0436 & 0.0530 & 0.0000 & 0.0000 \\
\hline (low, old) & 100 & 681 & 0.1468 & 0.0000 & 0.0000 & 0.1468 & 0.1733 & 0.0000 & 0.0000 \\
\hline (high, young) & 153 & 1385 & 0.1105 & 0.0000 & 0.0000 & 0.1105 & 0.1278 & 0.0000 & 0.0000 \\
\hline (high, old) & 278 & 1021 & 0.2723 & 0.0000 & 0.0411 & 0.2723 & 0.2997 & 0.0000 & 0.1509 \\
\hline Global & 610 & 4897 & 0.1246 & 0.0338 & 0.0830 & 0.4718 & 0.4993 & 0.0879 & 0.1758 \\
\hline
\end{tabular}

ald: age $\geq 40$ years; young: age $<40$

${ }^{\mathrm{b}} B M I$ body mass index; high: $\mathrm{BMI} \geq 25 \mathrm{~kg} / \mathrm{m}^{2}$; low: $\mathrm{BMI}<25$

${ }^{C} L C L$ lower confidence limit for the lower bound, $L B$ lower bound, $U B$ upper bound, $U C L$ upper confidence limit for the upper bound

for the cumulative completion risk of the specific class $=i, j$ sufficient-cause interaction (which they called 'weak' sufficient-cause interaction). Using the notations of this paper, their bound is

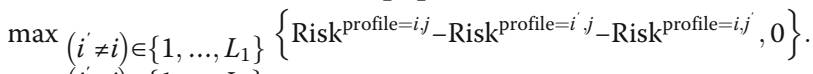
$\left(j^{\prime} \neq j\right) \in\left\{1, \ldots, L_{2}\right\}$

Additional file 4 shows we can achieve a sharper lower bound.

In this paper, the lower bound formulae also provide an avenue for testing of specific sufficient-cause interactions; if the bootstrapped 95\% lower confidence limits for a particular lower bound is greater than zero, then the corresponding sufficient-cause interaction is present. Alternatively, one can rely on the lower bound for the global sufficient-cause interaction; if its bootstrapped 95\% lower confidence limit is greater than zero, then some sufficient-cause interaction (between certain levels of the two factors) must be present. When both exposures are binary, such global test reduces to testing
PRISM $=1$ against PRISM $\neq 1$ in cohort studies [7], and RERI $=0$ against RERI $\neq 0$ in case-control studies.

The assumption of no confounding is a strong one. To alleviate the problem, the data can be stratified by the confounders (if these are identified and measured in the study) and separate bounds set on sufficient-cause interactions using the proposed formulae in this paper for each of the resulting strata. Further work is warranted to develop stratified bounding methods for sufficient-cause interactions when the total number of strata is large and the average stratum size is small (the sparse-data scenario) and when some of the stratifying variables also interact with the two exposures of concern (sufficientcause interactions involving more than two variables).

\section{Conclusions}

The study provides bounding formulae for sufficientcause interactions under the assumption of no redundancy. The bounds are sharp and sharper than previous

Table 2 Bounds on sufficient-cause interactions in a case-control study on lung cancer risk (Example 2)

\begin{tabular}{|c|c|c|c|c|c|}
\hline & \multirow{2}{*}{$\begin{array}{l}\text { Case } \\
\text { Number }\end{array}$} & \multirow{2}{*}{$\begin{array}{l}\text { Control } \\
\text { Number }\end{array}$} & \multirow{2}{*}{$\begin{array}{l}\text { Case- } \\
\text { Control } \\
\text { Odds }\end{array}$} & \multicolumn{2}{|c|}{ Relative prevalence of sufficient-cause interaction } \\
\hline & & & & $\overline{95 \% L^{2} L^{c}}$ & $\mathrm{LB}^{\mathrm{C}}$ \\
\hline \multicolumn{6}{|c|}{ Specific $\left(A D P R T^{\mathrm{a}}, X R C C 1^{\mathrm{b}}\right)$} \\
\hline (ValNal, Arg/Arg) & 157 & 186 & 0.8441 & 0.0000 & 0.0000 \\
\hline (ValNal, Arg/G/n) & 124 & 142 & 0.8732 & 0.0000 & 0.0000 \\
\hline (ValNal, Gln/Gln) & 26 & 31 & 0.8387 & 0.0000 & 0.0000 \\
\hline (Val/Ala, Arg/Arg) & 273 & 286 & 0.9545 & 0.0000 & 0.0000 \\
\hline (Val/Ala, Arg/Gln) & 180 & 183 & 0.9836 & 0.0000 & 0.0000 \\
\hline (Val/Ala, Gln/G/n) & 56 & 53 & 1.0566 & 0.0000 & 0.0000 \\
\hline (Ala/Ala, Arg/Arg) & 105 & 77 & 1.3636 & 0.0000 & 0.0000 \\
\hline (Ala/Ala, Arg/Gln) & 59 & 55 & 1.0727 & 0.0000 & 0.0000 \\
\hline (Ala/Ala, Gln/Gln) & 20 & 5 & 4.0000 & 0.0000 & 0.5221 \\
\hline Global & 1000 & 1018 & 0.9823 & 0.0784 & 0.2471 \\
\hline
\end{tabular}

${ }^{\mathrm{a}} A D P R T$ : adenosine diphosphate ribosyltransferase

${ }^{b} X R C C 1$ : $X$-ray repair cross-complementing group 1

${ }^{c} L C L$ lower confidence limit for the lower bound, $L B$ lower bound 
bounds. Sufficient-cause interactions cannot be estimated but can be quantified using the bounds presented in this study.

\section{Additional files}

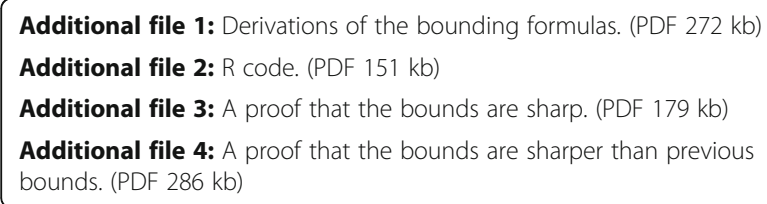

\section{Abbreviations}

ADPRT: Adenosine diphosphate ribosyltransferase; BMI: Body mass index; LB: Lower bound; PRISM: Peril ratio index of synergy based on multiplicativity; RERI: Relative excess risk due to interaction; RP: Relative prevalence; UB: Upper bound; XRCC1: X-ray repair cross-complementing group 1

\section{Acknowledgements}

Not applicable.

\section{Funding}

This paper is partly supported by grants from Ministry of Science and Technology, Taiwan (MOST 105-2314-B-002-049-MY3). No additional external funding received for this study. The funders had no role in study design, data collection and analysis, decision to publish, or preparation of the manuscript.

\section{Availability of data and materials}

The dataset supporting the conclusions of this article is included within the article and the Additional files.

\section{Author' contributions}

This is a single-authorship paper by WCL.

\section{Competing interests}

The author declares that he has no competing interests.

\section{Consent for publication}

Not applicable.

Ethics approval and consent to participate

Not applicable.

\section{Publisher's Note}

Springer Nature remains neutral with regard to jurisdictional claims in published maps and institutional affiliations.

Received: 29 November 2016 Accepted: 12 April 2017

Published online: 21 April 2017

\section{References}

1. VanderWeele TJ, Robins JM. The identification of synergism in the sufficientcomponent cause framework. Epidemiology. 2007;18:329-39.

2. VanderWeele TJ, Robins JM. Empirical and counterfactual conditions for sufficient cause interactions. Biometrika. 2008;95:49-61.

3. VanderWeele TJ. Sufficient cause interactions and statistical interactions. Epidemiology. 2009;20:6-13.

4. VanderWeele TJ. Sufficient cause interactions for categorical and ordinal exposures with three levels. Biometrika. 2010;97(3):647-59.

5. VanderWeele TJ, Knol MJ. Remarks on antagonism. Am J Epidemiol. 2011; 173:1140-7.

6. Lee WC. Testing synergisms in a no-redundancy sufficient-cause rate model. Epidemiology. 2013;24(1):174-5.

7. Lee WC. Assessing causal mechanistic interactions: a peril ratio index of synergy based on multiplicativity. PLoS ONE. 2013;8(6):e67424.

8. Lee WC. Estimation of a common effect parameter from follow-up data when there is no mechanistic interaction. PLoS ONE. 2014;9:e86374.
9. Lin JH, Lee WC. Testing for mechanistic interactions in long-term follow-up studies. PLOS ONE. 2015;10:e0121638.

10. Lee WC. Testing for sufficient-cause gene-environment interactions under independence and Hardy-Weinberg equilibrium assumptions. Am J Epidemiol. 2015;182(1):9-16.

11. Lee WC. Excess relative risk as an effect measure in case-control studies of rare diseases. PLoS ONE. 2015;10(4):e0121141.

12. Rothman KJ. Causes. Am J Epidemiol. 1976;104:587-92.

13. Rothman KJ, Greenland S, Lash TL, editors. Modern Epidemiology. 3rd ed. Philadelphia: Lippincott; 2008.

14. Greenland S, Brumback B. An overview of relations among causal modelling methods. Int J Epidemiol. 2002;31(5):1030-7.

15. Liao SF, Lee WC. Weighing the causal pies in case-control studies. Ann Epidemiol. 2010;20(7):568-73.

16. Suzuki E, Yamamoto $E$, Tsuda T. On the link between sufficient-cause model and potential-outcome model. Epidemiology. 2011;22(1):131-2.

17. Suzuki E, Yamamoto E, Tsuda T. On the relations between excess fraction, attributable fraction, and etiologic fraction. Am J Epidemiol. 2012;175(6):567-75.

18. Lee WC. Completion potentials of sufficient component causes. Epidemiology. 2012;23(3):446-53.

19. Gatto NM, Campbell UB. Redundant causation from a sufficient cause perspective. Epidemiol Perspect Innov. 2010;7:5

20. Sjölander A, Lee W, Källberg H, Pawitan Y. Bounds on sufficient-cause interaction. Eur J Epidemiol. 2014;29:813-20.

21. Zou GY. On the estimation of additive interaction by use of the four-by-two table and beyond. Am J Epidemiol. 2008;168:212-24.

22. Zhang X, Miao X, Liang G, Hao B, Wang Y, Tan W, Li Y, Guo Y, He F, Wei Q, Lin D. Polymorphisms in DNA base excision repair genes ADPRT and XRCC1 and risk of lung cancer. Cancer Res. 2005;65:722-6.

\section{Submit your next manuscript to BioMed Central and we will help you at every step:}

- We accept pre-submission inquiries

- Our selector tool helps you to find the most relevant journal

- We provide round the clock customer support

- Convenient online submission

- Thorough peer review

- Inclusion in PubMed and all major indexing services

- Maximum visibility for your research

Submit your manuscript at www.biomedcentral.com/submit
Biomed Central 